\title{
Tratamento de fratura nasal pediátrica: relato de caso clínico
}

\author{
Pediatric nasal fracture treatment: clinical case \\ Tratamiento de fratura nasal pediátrica: reporte de caso \\ Júlio Leite de ARAÚJo-JÚNIOR ${ }^{1}$ \\ Elma Mariana Verçosa de MELO-SILVA ${ }^{1}$ \\ Anderson Maikon de SOUZA-SANTOS ${ }^{1}$ \\ Tiburtino José de LIMA-NETO' \\ Murilo Quintão dos SANTOS ${ }^{1}$ \\ Eduardo DIAS-RIBEIRO ${ }^{2}$ \\ ${ }^{I}$ Residentes de Cirurgia e Traumatologia Buco-Maxilo-Facial pela Universidade Federal da Paraíba, UFPB, 58051-900 João Pessoa, Paraíba, Brasil \\ ${ }^{2}$ Professor do Programa de Residência em Cirurgia e Traumatologia Buco-Maxilo-Facial da UFPB, 58051-900 João Pessoa, Paraíba, Brasil
}

\begin{abstract}
Resumo
Introdução: Os ossos nasais são os mais proeminentes do esqueleto facial, tornando esses os mais frequentes nas fraturas faciais, sendo o terceiro osso mais comumente fraturado do esqueleto humano. Objetivo: Apresentar um relato de caso de fratura nasal em um paciente pediatrico tratado com redução incruenta. Método: Estudo descritivo com um paciente que apresentou diagnóstico clínico/imaginológico de fratura nasal. Conclusão: O tratamento através de redução incluenta mostrou-se adequado em pacientes pediatricos. A ocorrência de traumatismos e lesões associadas a fraturas nasais reforça a importância de uma abordagem multidisciplinar.
\end{abstract}

Descritores: Fraturas Ósseas; Osso Nasal; Traumatismos Faciais.

\section{Abstract}

Introduction: Nasal bones are the most prominent of the facial skeleton, making them the most frequent in facial fractures, being the third most commonly fractured bone of the human skeleton. Purpose: To present a case report of a nasal fracture in a pediatric patient treated with a bloodless reduction. Method: Descriptive study with a patient who presented clinical / imaging diagnosis of nasal fracture. Conclusion: Treatment through reduction included was adequate in pediatric patients. The occurrence of trauma and lesions associated with nasal fractures reinforces the importance of a multidisciplinary approach.

Descriptors: Fractures, bone; Nasal Bone; Facial Injuries.

\section{Resumen}

Introducción: Los huesos nasales son los más prominentes del esqueleto facial, haciéndolos los más frecuentes en las fracturas faciales, siendo el tercer hueso más comúnmente fracturado del esqueleto humano. Objetivo: Presentar un relato de caso de fractura nasal en un paciente pediatrico tratado con reducción incruenta. Método: Estudio descriptivo con un paciente que presentó diagnóstico clínico / imaginológico de fractura nasal. Conclusión: El tratamiento a través de la reducción incluye se muestra apropiado en pacinas pediátricas. La ocurrencia de traumatismos y lesiones asociadas a fracturas nasales refuerza la importancia de un enfoque multidisciplinario.

Descriptores: Fracturas Óseas; Hueso Nasal; Traumatismos Faciales.

\section{INTRODUÇÃO}

Os ossos nasais são os mais proeminentes do esqueleto facial, tornando esses os mais frequentes nas fraturas faciais, sendo o terceiro osso mais comumente fraturado do esqueleto humano. Sendo sua espessura reduzida e sua posição proeminente na face o principal motivo das fraturas. Onde, pequenas forças podem fraturar os ossos nasais, enquanto forças maiores podem causar fraturas cominutivas. Agressões físicas, quedas de própria altura, acidentes motociclísticos, automobilístico, esportivos são os fatores etiológicos mais implicados no trauma aos ossos nasais ${ }^{1,2}$.

As fraturas do esqueleto facial pediátricas são raras comparadas com as fraturas do esqueleto facial adultas. Estudos retrospectivos de trauma facial, envolvendo adultos e crianças, demonstraram que menos de $15 \%$ dessas fraturas ocorrem em pessoas mais jovens, menos de 16 anos de idade, onde, menos de $1 \%$ de todas as fraturas faciais ocorrem em crianças menores de 5 anos de idade ${ }^{3}$.

Os ossos nasais são unidos na linha média facial formando a porção óssea da arquitetura nasal, sendo mais espessos superiormente e mais finos inferiormente. O suporte superior e lateral provém dos processos nasais do osso frontal e da maxila respectivamente. A arquitetura do nariz é dada também pela anatomia dos tecidos moles, onde a porção cartilaginosa, composta por duas cartilagens laterais superiores contínuas com o osso nasal e duas cartilagens laterais inferiores conformam a região lateral anterior. Pequenos fragmentos de cartilagem, cartilagens sesamoides, completam a estrutura nasal cartilaginosa externa ${ }^{4,5}$.

As fraturas nasais são de diagnóstico clínico, sendo as radiografias complementares para esses diagnósticos, onde a tomografia computadorizada é um exame de imagem padrão ouro para fraturas faciais em geral, no entanto devido ao elevado custo, exposição à radiação e à falta de impacto no manejo do trauma, não deve ser rotineiramente empregada, a menos que se suspeite de fraturas associadas ${ }^{6,7}$.

O tratamento das fraturas nasais é geralmente realizado através da redução fechada. A literatura, de uma forma geral, apresenta bons resultados. Nesse sentido, este trabalho tem por objetivo apresentar um caso de fratura nasal pediátrica tratado por redução incruenta sob anestesia geral.

\section{CASO CLÍNICO}

Paciente do gênero masculino, 13 anos de idade, foi encaminhado para o Hospital Estadual de Emergência e Trauma Senador Humberto Lucena em João Pessoa, Paraíba, Brasil após acidente desportivo. Inicialmente o paciente foi encaminhado para o setor 
pediátrico para avaliação e estabilização do quadro, inicialmente o paciente se apresentou consciente, orientado, eupnéico, em bom estado geral. A pediatra de plantão solicitou a avaliação da Cirurgia Buco-Maxilo-Facial. Ao exame físico foi observado edema em região de ossos próprios do nariz (OPN) como pode ser visto na vista frontal, crânio-caudal e caudo-cranial evidenciando o afundamento da parede nasal lateral direita, obstrução nasal, movimentos oculares e acuidade visual mantida, boa abertura bucal e oclusão estável (Figuras 1, 2 e 3). Os exames radiológicos foram solicitados, onde foi observado um traço de fratura em OPN, como pode ser visto nos exames radiográficos perfil de face e incidência de Waters (Figuras 4 e 5). O clínico associado ao exame complementar levou o cirurgião a dar o diagnóstico de fratura de OPN, devido ao edema que estava estabelecido o paciente foi liberado e o retorno marcado para 7 dias.

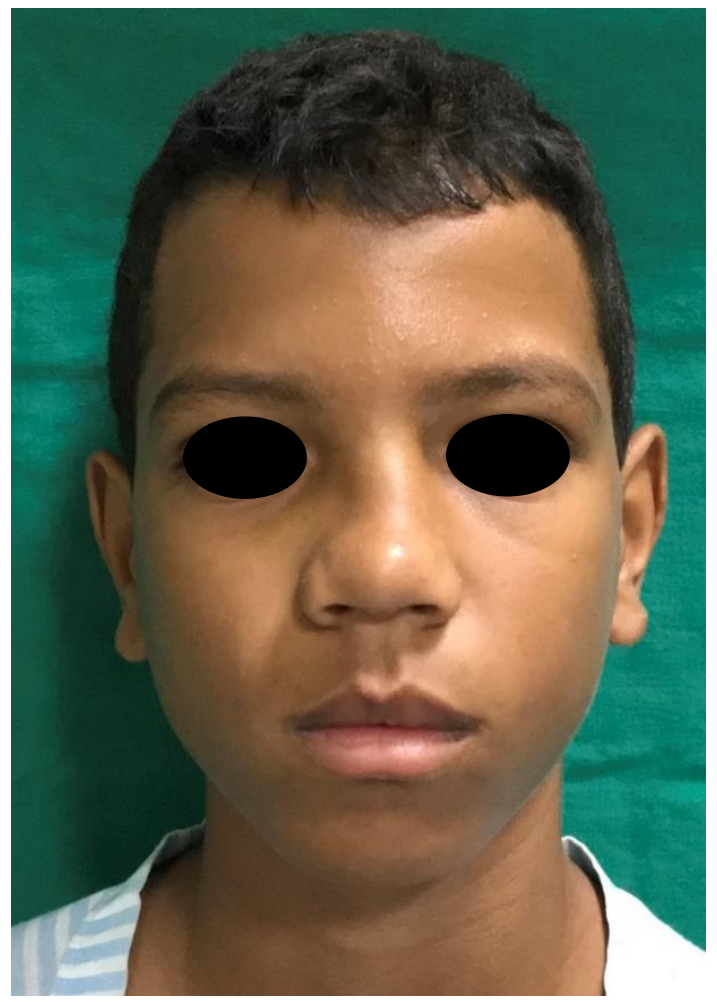

Figura 1: Vista frontal evidenciando edema nasal.

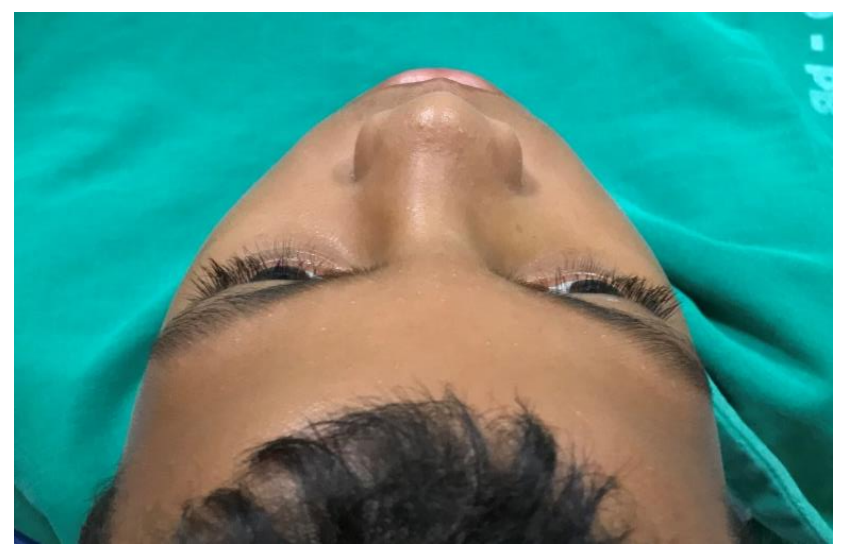

Figura 2: Vista craniocaudal evidenciando edema nasal e afundamento da parede nasal lateral direita.

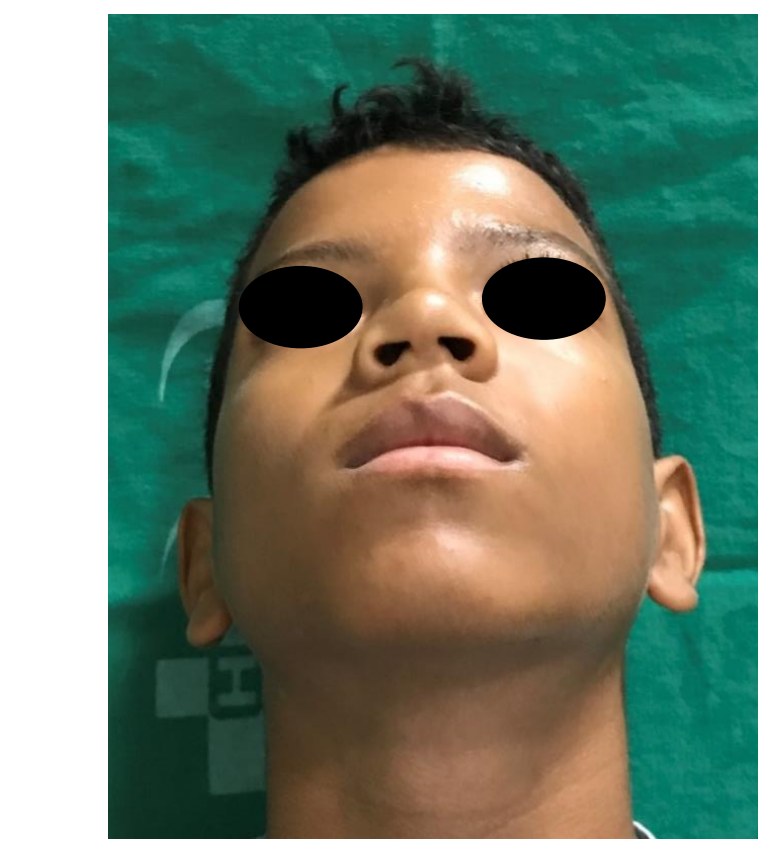

Figura 3: Vista caudocranial evidenciando edema nasal e afundamento da parede nasal lateral direita.

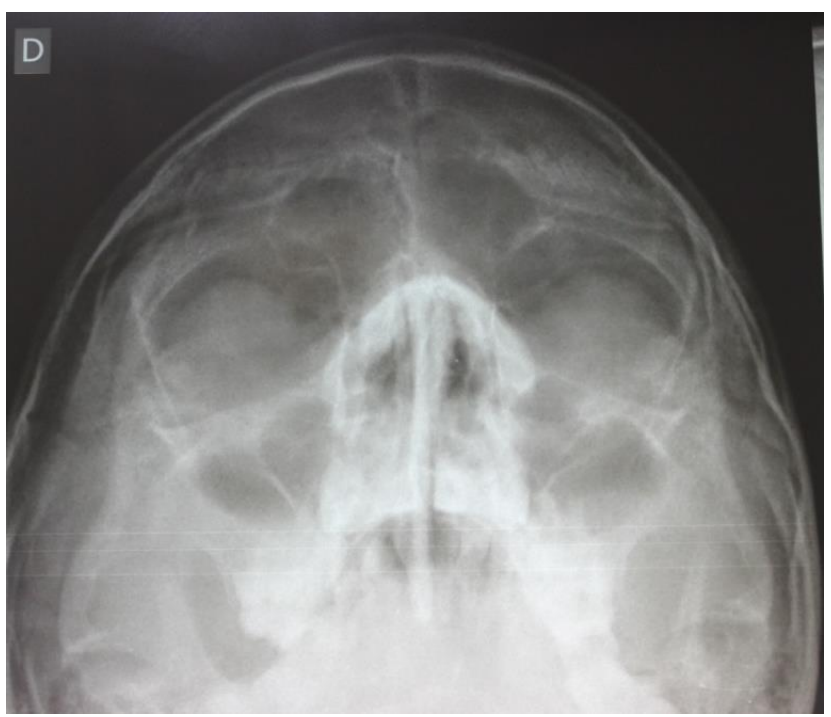

Figura 4: Incidência de Waters evidenciando traço de fratura em OPN.

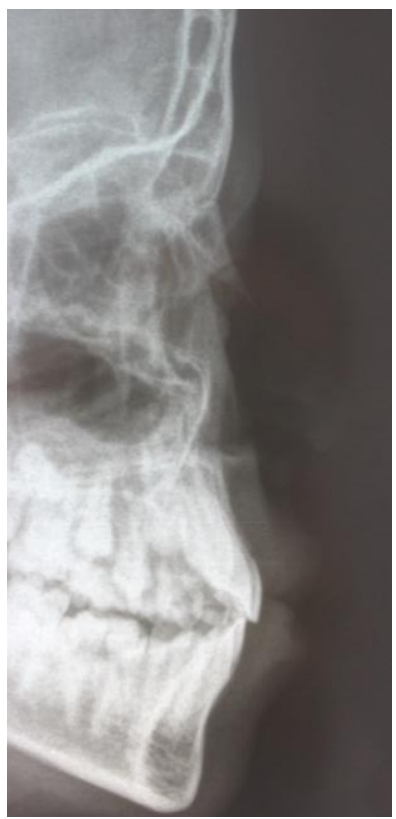

Figura 5: Radiografia perfil de face evidenciando traço de fratura em OPN. 
No retorno o paciente apresentou regressão total do edema e observou-se o afundamento da parede lateral dos ossos nasais e obstrução nasal foi relatada, desse modo, foi indicado o tratamento cirúrgico sob anestesia geral. $\mathrm{O}$ paciente foi encaminhado ao centro cirúrgico do hospital, submetido a anestesia geral por via orotraqueal, os campos cirúrgicos foram posicionados, a redução foi feita com o uso das pinças para redução nasal, Asch e Walshamn, após a redução foi feito um tamponamento nasal anterior para estabilização da fratura e observado a redução da fratura através de radiografia perfil de face e incidência de Waters (Figuras 6 e 7). O paciente teve alta com antibiótico, analgésico e anti-inflamatório e retorno marcado para remoção do tampão após três dias. No acompanhamento pós-operatório o tampão foi removido e o paciente acompanhado semanalmente até a regressão total do edema. Após 1 mês observouse regressão do edema, melhora da obstrução nasal e contorno facial reestabelecido (Figuras 8 e 9).

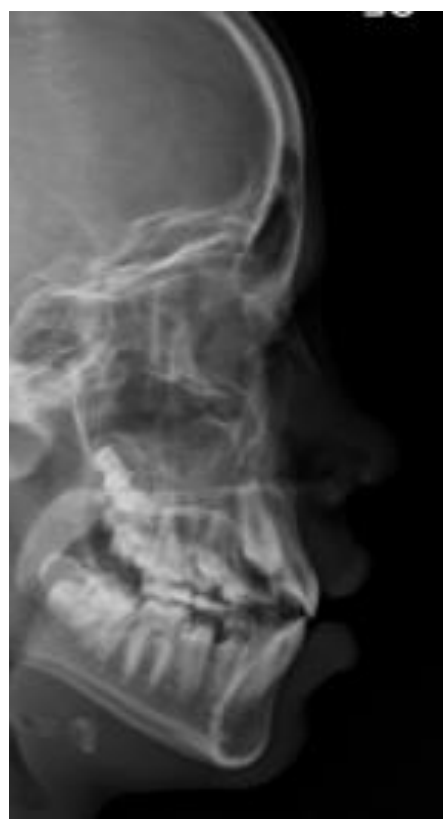

Figura 6: Redução da fratura vista através de radiografia de perfil de face.

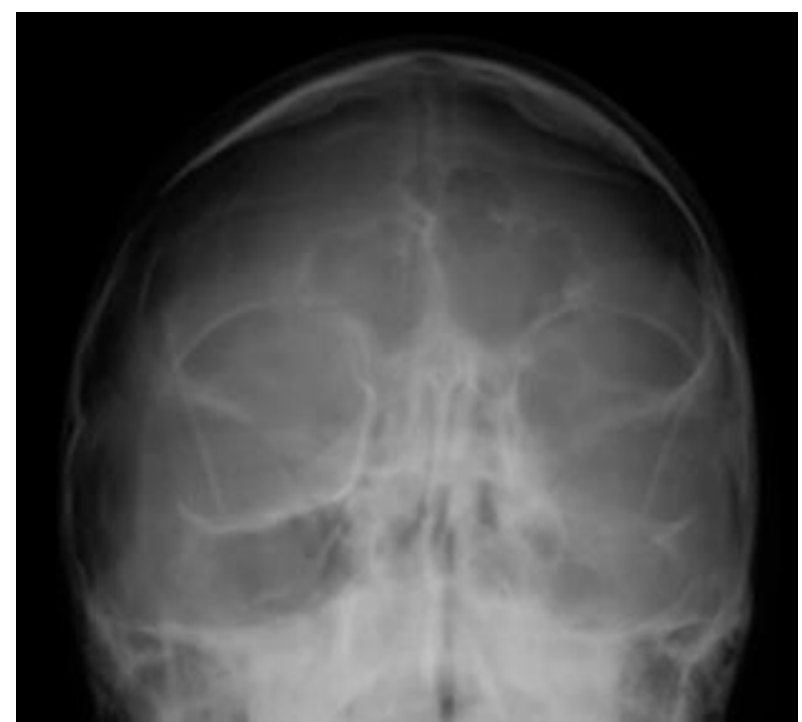

Figura 7: Redução da fratura através de incidência de Waters.

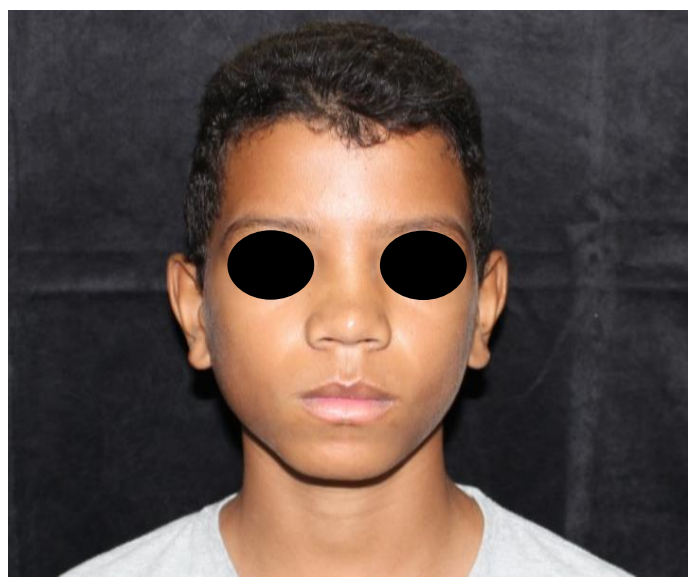

Figura 8: Regressão do edema e contorno facial reestabelecido após 30 dias de redução de fratura de OPN vista em posição frontal.

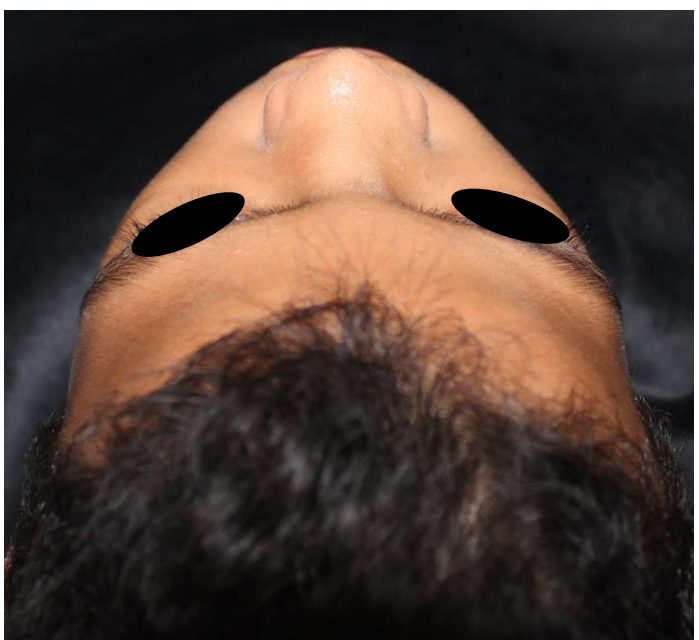

Figura 9: Regressão do edema e contorno facial reestabelecido após 30 dias de redução de fratura de OPN vista em posição craniocaudal.

\section{DISCUSSÃO}

Dificuldades no tratamento de fratura em crianças em comparação com os adultos possuem algumas diferenças devido à necessidade do crescimento facial. Considerando que absoluta redução e fixação está indicada em adultos, o cuidado com a mínima manipulação do esqueleto facial em crianças é mandatório para prevenir anormalidades de crescimento. Além disso, descolamento periosteal pode afetar o crescimento ósseo e o tratamento necessário para uma completa reconstrução pode afetar negativamente o crescimento $\mathrm{e}$ desenvolvimento da face ${ }^{8}$.

Desse modo, as fraturas nasais são a fratura facial mais comum e a maioria deles são tratadas com sucesso com redução fechada. Contudo, em pacientes pediátricos, parece difícil reduzir as fraturas nasais com precisão porque o osso nasal pediátrico é muito pequeno para confirmar a redução ${ }^{9}$. Desse modo, o paciente relatado foi submetido ao tratamento cirúrgico fechado, com a utilização de pinças para redução nasal, Asch e Walshamn, observando-se resultado satisfatório ${ }^{9}$. 
Os ossos faciais infantis são protegidos pelo baixo impacto devido a relação de volume que permite ao crânio, ao invés da face, absorver a maior parte do impacto traumático. Além disso, esses ossos são mais resistentes em crianças devido a sua maior elasticidade, falta de pneumatização sinusal, paredes ósseas mais espessas que são envolvidas pelo tecido adiposo e estabilização da mandíbula e maxila por dentes não irrompidos ${ }^{3}$.

Murphy et al. ${ }^{10}$ categorizaram o tempo para a cirurgia como: precoce ( 7 dias) e tardio ( $>7$ dias). Em uma amostra de 35 pacientes observaram que o tratamento tardio não afetou significativamente $o$ resultado cirúrgico em longo prazo em pacientes pediátricos. Tal fato pode ser atribuído aos ossos mais elásticos e macios, tecidos relativamente mais abundantes em crianças quando comparadas aos adultos. Em concordância com o nosso caso, em que o paciente foi encaminhado ao centro cirúrgico após 7 dias de ocorrido o trauma, categorizado-se no tratamento tardio, assim observado-se uma reposta adequada ao tratamento nesse período ${ }^{10}$.

Em análise de 809 fraturas de ossos faciais em população pediátrica e adolescente, o osso nasal foi o mais comumente fraturado ${ }^{9}$. Como nos adultos, as razões para isso podem ser sua posição central proeminente e a delicadeza do osso nasal ${ }^{9}$. Esses pacientes apresentaram desse modo uma baixa frequência de complicações após o período de um ano $^{9,10}$. Ocorrendo do mesmo modo no pós-operatório do paciente deste relato, tendo transcorrido sem complicações no período de um mês.

\section{CONCLUSÃO}

A ocorrência de traumatismos e lesões associadas a fraturas nasais reforça a importância de uma abordagem multidisciplinar, buscando $\mathrm{o}$ conforto do paciente no período pós-operatório. Desse modo, o tratamento através de redução incluenta mostrou-se adequado em pacientes pediatricos, devolvendo um bom resultado estético e funcional.

\section{REFERÊNCIAS}

1. Ma L, Shen SH, Hu P, Wu ZQ. The observation of curative effect on closed reduction of nasal bone fracture under ultrasound guidance: report of 38 cases. Zhonghua Er Bi Yan Hou Tou Jing Wai Ke Za Zhi. 2017;52(12):933-35.

2. Schoinohoriti O, Igoumenakis D, Rallis G. Fractures of the nasal bones: is external splinting really warranted? J Craniofac Surg. 2017;28(8):e760-e63.

3. Kang CM, Han DG. Correlation between Operation Result and Patient Satisfaction of Nasal Bone Fracture. Arch Craniofac Surg. 2017;18(1):25-9.
4. Kyung H, Choi JI, Song SH, Oh SH, Kang N. Comparison of postoperative outcomes between monitored anesthesia care and general anesthesia in closed reduction of nasal fracture. J Craniofac Surg. 2018;29(2):286-88.

5. Nishioka H, Kondoh S, Yuzuriha S. Convex bone deformity after closed reduction of nasal bone fracture. J Plast Reconstr Aesthet Surg. 2018;71(1):85-9.

6. Lu GN, Humphrey CD, Kriet JD. Correction of Nasal Fractures. Facial Plast Surg Clin North Am. 2017;25(4):537-546.

7. Kim SW, Park B, Lee TG, Kim JY. Olfactory Dysfunction in Nasal Bone Fracture. Arch Craniofac Surg. 2017;18(2):92-6.

8. Davidson J, Nickerson D, Nickerson B. Zygomatic fractures: comparison of methods of internal fixation. Plast Reconstr Surg. 1990;86(1):25-32.

9. Yabe T, Tsuda T, Hirose S, Ozawa T. Comparison of pediatric and adult nasal fractures. J Craniofac Surg. 2012;23(5):1364-6.

10.Murphy RX Jr, Birmingham KL, Okunski WJ, Wasser TE. Influence of restraining devices on patterns of pediatric facial trauma in motor vehicle collisions. Plast Reconstr Surg. 2001;107(1):34-7.

\section{CONFLITO DE INTERESSES}

Os autores declaram não haver conflitos de interesse.

\section{AUTOR PARA CORRESPONDENCIA}

Júlio Leite de Araújo Júnior

juniorleitearaujo@hotmail.com

Submetido em 27/05/2018

Aceito em 27/06/2018 